\title{
Feeding Threshold for Predators Stabilizes Predator-Prey Systems
}

\author{
D. Bontje $^{1 *}$, B.W. Kooi ${ }^{1}$, G.A.K. van Voorn ${ }^{2}$ and S.A.L.M Kooijman ${ }^{1}$ \\ ${ }^{1}$ Department of Theoretical Biology, Vrije Universiteit, \\ De Boelelaan 1085, 1081HV Amsterdam, The Netherlands \\ ${ }^{2}$ Biometris, Wageningen Universiteit \& Research, \\ 6708PB Wageningen, The Netherlands
}

\begin{abstract}
Since Rosenzweig showed the destabilisation of exploited ecosystems, the so called Paradox of enrichment, several mechanisms have been proposed to resolve this paradox. In this paper we will show that a feeding threshold in the functional response for predators feeding on a prey population stabilizes the system and that there exists a minimum threshold value above which the predator-prey system is unconditionally stable with respect to enrichment. Two models are analysed, the first being the classical Rosenzweig-MacArthur (RM) model with an adapted Holling type-II functional response to include a feeding threshold. This mathematical model can be studied using analytical tools, which gives insight into the mathematical properties of the two dimensional ODE system and reveals underlying stabilisation mechanisms. The second model is a mass-balance (MB) model for a predator-prey-nutrient system with complete recycling of the nutrient in a closed environment. In this model a feeding threshold is also taken into account for the predator-prey trophic interaction. Numerical bifurcation analysis is performed on both models. Analysis results are compared between models and are discussed in relation to the analytical analysis of the classical RM model. Experimental data from the literature of a closed system with ciliates, algae and a limiting nutrient are used to estimate parameters for the MB model. This microbial system forms the bottom trophic levels of aquatic ecosystems and therefore a complete overview of its dynamics is essential for understanding aquatic ecosystem dynamics.
\end{abstract}

Key words: algal-ciliate experiments $\cdot$ feeding threshold $\cdot$ mass-balance $\cdot$ paradox of enrichment . strong stability

AMS 2000 subject classification: 92D40, 92B05, 34K18

\footnotetext{
*Corresponding author. E-mail: daniel.bontje@falw.vu.nl
} 


\section{Introduction}

In the classical Rosenzweig-MacArthur (RM) model a bifurcation occurs when the carrying capacity of the prey reaches high values [27]. Then the steady-state becomes unstable and a stable periodic solution originates. This transition is the Hopf bifurcation point. For carrying capacity values above the Hopf bifurcation point, the amplitude of the oscillatory dynamics increases. As a result the minimum value for the prey population becomes very low and extinction due to stochastic fluctuations becomes likely. This phenomenon is called the "paradox of enrichment".

Kirk (1998) suggested that the solution to the paradox of enrichment lies in the fact that many biological models show a lack of biological detail. Indeed, many biological models that include more detail than the RM model seem to resolve the paradox by preventing destabilisation under nutrient enrichment. Among the underlying mechanisms are the division of the prey-population into two subpopulations one accessible, vulnerable or edible and one inaccessible, invulnerable or inedible [15, 2], self-limitation of the prey [12], predator-induced defence mechanisms in the prey population [32], dormancy of the predators [16] and spatial heterogeneity [11, 29, 25]. Whether these mechanisms do cause stability depends on model specifics, as for example, adaptive defence of the prey can be both stabilizing [32] and destabilizing [1].

The stability of ecological systems is extremely sensitive to the exact form of the functional response that models the trophic interactions [10]. In [31] it is shown that the paradox of enrichment can indeed be avoided by using the functional response proposed by Beddington (1975) and DeAngelis et al. (1975) that takes intraspecific interference between predators into account.

In this paper we evaluate the effects of a feeding threshold on ecosystem stability and/or persistence. In light of the comments made in [9], we define stability in the mathematical sense as the stability of steady states with respect to small perturbations. We use the definitions for stability as provided in [31], where weak stability is defined as the delayed occurrence of a Hopf bifurcation and strong stability as the disappearance of the Hopf bifurcation for all states of enrichment.

Here the Holling type-II functional response is adapted by the introduction of a fixed threshold for the prey population below which all prey individuals are not at risk of predation. For example because at low food densities the predator stops searching $[22,28]$ or the prey hides in spatial separated areas called refuges. Different types and causes of refuges are discussed in [4]. This type of functional response is used in aquatic ecosystem modelling: for instance in the AQUATOX program that predicts the fate of various pollutants, such as nutrients and organic chemicals, and their effects on the ecosystem, including fish, invertebrates, and aquatic plants [23, 24].

The main goal of this paper is to study how the inclusion of a feeding threshold in the Holling type-II functional response for predator-prey interaction affects the behaviour of two different models which each simulate a nutrient-algae-ciliate microbial ecosystem in a spatial homogeneous and closed environment. One model is based on a mass-balance (MB) formulation which is derived from first principles and includes complete nutrient recycling [7, 14] and the Holling type-II functional responses are used for the nutrient-algae interaction and the algae-ciliate interaction, only the latter includes a feeding threshold. The other model is the classical RM model [27] which only implicitly includes a nutrient and does not include mass-balance.

Before starting the analysis of the effect of a feeding threshold on stability in our MB model, we 
first study the simpler RM model [27] as symbolic algebra programs allow for a complete analysis of the model. Then we continue with a numerical bifurcation analysis of the MB model. For both models we study the effect of the feeding threshold on the paradox of enrichment by bifurcating both the threshold value and the nutrient load of system. Important is the minimal nutrient load of the system at which the algae can invade the system, then a transcritical bifurcation (TC) occurs. The second TC bifurcation occurs when the nutrient load is high enough to support a producer population that is sufficiently dense enough to allow predator invasion. This last TC marks the region where the prey and predator can coexist. At even higher nutrient loads a Hopf bifurcation marks regions where the system shows oscillatory dynamics [13]. We will show that the long-term dynamic behaviour of the RM and MB systems appear to be similar and are qualitatively even the same.

To illustrate the existence of a feeding threshold, we apply the MB model on experimental data consisting of dynamic growth curves of prey and predator from [33]. During batch experiments the flagellated algal species Cryptomonas sp. was preyed upon by one of three ciliate species, either Balanion planctonicum, Urotricha furcata or Urotricha farcta. All species are fresh water micro-organisms.

\section{Formulation of the model}

We study a spatial homogeneous and closed system with a predator, $Z$ and a prey population, $P$, consuming a limiting nutrient, $N$. Other nutrients, including sunlight, are not limiting. The mortality rate of each population is denoted by $d_{P}$ for the prey and $d_{Z}$ for the predator. Table 1 lists all used variables and parameters together with a short description and units.

The interactions between the trophic levels are modelled with a Holling type-II functional response. Parameters for the prey-nutrient interaction are the searching rate $v_{N}$ and the nutrient handling time $h_{N}$ and similar for the predator-prey interaction are the searching rate $v_{P}$ and the prey handling time $h_{P}$. We implement a numerical feeding threshold, $\tau$, by using a maximumfunction denoted with $(P-\tau)_{+}=\max (P-\tau, 0)$, which either yields zero or a positive value when $P>\tau$.

The predator population digests its prey only partly. The conversion efficiency of prey biomass into predator biomass is denoted by the yield factor $y_{P Z}$. The unusable part of the food is ejected into the environment as faeces. This excreted material together with dead material forms detritus and is decomposed instantaneously and this gives complete recycling of the nutrient. The above leads to the following ODE system

$$
\begin{aligned}
\frac{d N}{d t} & =-P \frac{v_{N} N}{1+v_{N} h_{N} N}+\left(1-y_{P Z}\right) Z \frac{v_{P}(P-\tau)_{+}}{1+v_{P} h_{P}(P-\tau)_{+}}+d_{P} P+d_{Z} Z . \\
\frac{d P}{d t} & =P\left(\frac{v_{N} N}{1+v_{N} h_{N} N}-d_{P}\right)-Z \frac{v_{P}(P-\tau)_{+}}{1+v_{P} h_{P}(P-\tau)_{+}}
\end{aligned}
$$




$$
\begin{aligned}
\frac{d Z}{d t} & =Z\left(y_{P Z} \frac{v_{P}(P-\tau)_{+}}{1+v_{P} h_{P}(P-\tau)_{+}}-d_{Z}\right), \\
I_{N P} & =1 / h_{N} \quad ; \quad K_{N P}=1 /\left(v_{N} h_{N}\right) ; \quad I_{P Z}=1 / h_{P} \quad ; \quad K_{P Z}=1 /\left(v_{P} h_{P}\right) .
\end{aligned}
$$

The Michaelis-Menten parameters, being the maximum ingestion rates $\left(I_{N P}\right.$ and $\left.I_{P Z}\right)$ and the nutrient half-saturation concentrations $\left(K_{N P}\right.$ and $\left.K_{P Z}\right)$, are compound parameters of the searching rate $v$ and the handling time $h$. The last three terms of Eq. (2.1a) are the terms for instantaneous and complete degradation of faeces and dead organisms. In the Appendix we show how a system of limiting nutrient, producer, predator, bacteria and detritus can be reduced to the system above while obeying mass-conservation.

Because of this recycling and mass-conservation, we can reduce the three dimensional system to an equivalent two dimensional system. Let $N_{T}$ denote the nutrient load or more precise the total amount of limiting nutrient in the closed system formed by the biota $P, Z$ and the abiotic environment $N$ defined as

$$
N_{T}=N(t)+P(t)+Z(t) .
$$

Adding the three equations of Eq. (2.1) shows that

$$
\frac{d N_{T}}{d t}=0 \quad \text { and } \quad N_{T}=N(0)+P(0)+Z(0)
$$

that is, $N_{T}$ is a constant. The resulting two dimensional system becomes

$$
\begin{aligned}
\frac{d P}{d t} & =P\left(f(N)-d_{P}\right)-Z f(P) \\
\frac{d Z}{d t} & =Z\left(y_{P Z} f(P)-d_{Z}\right) \\
f(N) & =\frac{v_{N}\left(N_{T}-P-Z\right)}{1+v_{N} h_{N}\left(N_{T}-P-Z\right)} \quad ; \quad f(P)=I_{P Z} \frac{(P-\tau)_{+}}{K_{P Z}+(P-\tau)_{+}} .
\end{aligned}
$$

To have a mathematically well-posed problem we require for the initial value conditions that $N(0)=N_{T}-P(0)-Z(0)>0$.

When taking $h_{N}=0, f(N)$ becomes a linear functional response, this yields:

$$
\begin{aligned}
& \frac{d P}{d t}=r P\left(1-\frac{P+\alpha Z}{K}\right)-Z f(P), \\
& \frac{d Z}{d t}=Z\left(y_{P Z} f(P)-d_{Z}\right),
\end{aligned}
$$

where

$$
K=N_{T}-\frac{d_{P}}{v_{N}} \quad ; \quad r=v_{N} K=v_{N} N_{T}-d_{P} \quad ; \quad \alpha \in\{0,1\}
$$


In absence of the predator the prey population grows logistically. Note that the intrinsic growth rate $r$ and carrying capacity $K$ are both expressed as a function of $N_{T}$ using parameters from Eq. (2.1).

With $\alpha=1$ in Eq. (2.4a) we obtain a mass-balance model for both $P$ and $Z$. Posteriorly the nutrient density $N(t)$ can be calculated using Eq. (2.2). The above ODE system with $\alpha=0$ is identical to the RM model, which in its usual form has $r$ and $K$ as independent constants. Due to our formulation, $r \propto K$. As will be shown in the next section, for $\tau=0$ the paradox of enrichment can still occur. When $\alpha=0$, then the term $Z / K$ is removed from Eq. (2.4a), leading to an ODEsystem without mass-conservation. Consequently, Eq. (2.2) for $N_{T}$ no longer holds and the value for variable $N$ can not be calculated.

We briefly repeat the main differences between the RM and MB model: the first has a nutrient handling time of zero and no mass-conservation, the latter has a non-zero nutrient handling time and respects mass-conservation. By setting $\alpha=0$ and $h_{N}=0$ one obtains the RM model from the MB model.

\section{Analysis of the models}

The two dimensional systems can be analysed with a stability analysis and by studying the zerogrowth isoclines. The stability properties of the resulting equilibria are derived using nonlinear dynamic system theory. When $\mathbf{J}$ denotes the Jacobian matrix evaluated at the equilibrium, its stability is directly determined by the sign of the eigenvalue of the real parts. For systems with two variables, the transcritical bifurcation, $\tau_{T C}\left(N_{T}\right)$, and the Hopf bifurcation curve, $\tau_{H}\left(N_{T}\right)$, are determined by $\operatorname{det} \mathbf{J}=0$ and trace $\mathbf{J}=0$, respectively.

\subsection{The Rosenzweig-MacArthur model}

For the RM model Eqs. (2.4) and depending on the parameter values, there is one equilibrium with no species, denoted by $E_{0}$, one with only the producer, $E_{1}$, and one with coexistence of the producer and predator, $E_{2}$. Using symbolic calculation software, such as Maple [18], we obtain algebraic expressions for the isoclines, the transcritical bifurcations and Hopf bifurcation codim-1 curves. These lengthy expression are not given here. However, the curves are shown in the left panels of Figure 1.

These panels show the long-term dynamic behaviour of the two populations without and with a feeding threshold, respectively $\tau=0$ and $\tau=0.1$. For low values of $N_{T}$ only the prey population exists. For larger $N_{T}$ both populations coexist stably. The transition is at the transcritical bifurcation point. Increasing $N_{T}$ further, the steady-state becomes unstable and a stable periodic solution originates. This transition is at the Hopf bifurcation point. For $N_{T}$ values above the Hopf bifurcation point, the amplitude of the oscillatory dynamics increases. As a result the minimum value for the prey population becomes very low and extinction due to stochastic fluctuations becomes likely. This phenomenon is called the "paradox of enrichment". 
Table 1: List of Symbols

\begin{tabular}{|c|c|c|}
\hline Symbol & Description & Units \\
\hline$\alpha$ & Eq. (2.4) with $\alpha=0$ forms the RM model. With $\alpha=1$ & $-/-$ \\
\hline & a model with a correct mass-balance would be formed & \\
\hline$C$ & ciliate (predator) density & \#cells $\mathrm{L}^{-1}$ \\
\hline$d_{P}$ & death rate prey & $\mathrm{d}^{-1}$ \\
\hline$d_{Z}$ & death rate predator & $\mathrm{d}^{-1}$ \\
\hline$F$ & flagellate (prey) density & \#cells $\mathrm{L}^{-1}$ \\
\hline$f(P)$ & Holling type-II functional response of $Z$ on $P$ & $\mathrm{~d}^{-1}$ \\
\hline$F_{0}(0)$ & initial flagellate density without ciliates present & \#cells $\mathrm{L}^{-1}$ \\
\hline$F_{C}(0)$ & initial flagellate density with ciliates present & \#cells $\mathrm{L}^{-1}$ \\
\hline$g(P)$ & prey zero-growth isocline for RM model & $\operatorname{mol~N~L}{ }^{-1}$ \\
\hline$h_{N}$ & nutrient handling time & $\mathrm{d}$ \\
\hline$h(P)$ & prey zero-growth isocline for MB model & $\operatorname{mol~N~L}{ }^{-1}$ \\
\hline$h_{P}$ & prey handling time & d \\
\hline$I_{F C}$ & maximum specific ingestion rate of prey biomass & prey predator ${ }^{-1} \mathrm{~h}^{-1}$ \\
\hline$I_{P Z}$ & maximum specific ingestion rate of prey biomass & $\operatorname{mol~N~mol~N} \mathrm{N}^{-1} \mathrm{~d}^{-1}$ \\
\hline$I_{N P}$ & maximum specific ingestion rate of limiting nutrient & $\operatorname{mol~N~mol~N}{ }^{-1} \mathrm{~d}^{-1}$ \\
\hline$K$ & prey's carrying capacity & $\operatorname{mol~N~L}{ }^{-1}$ \\
\hline$K_{N P}$ & nutrient half-saturation concentration for $P$ on $N$ & $\operatorname{mol~N~L}{ }^{-1}$ \\
\hline$K_{P Z}$ & nutrient half-saturation concentration for $Z$ on $P$ & $\operatorname{mol~N~L}{ }^{-1}$ \\
\hline$N$ & limiting nutrient concentration & $\operatorname{mol~N~L}{ }^{-1}$ \\
\hline$n_{C}$ & predator limiting nutrient content & $\mathrm{pmol} \mathrm{N} / \mathrm{cell}$ \\
\hline$n_{F}$ & prey limiting nutrient content & pmol N /cell \\
\hline$N_{T}$ & total amount of limiting nutrient in the system (nutrient load) & $\operatorname{mol~N~L}{ }^{-1}$ \\
\hline$P$ & prey biomass density & $\operatorname{mol~N~L}{ }^{-1}$ \\
\hline$r$ & prey percapita growth rate & $\mathrm{d}^{-1}$ \\
\hline$\tau$ & feeding threshold in units of prey biomass & $\operatorname{mol~N~L}{ }^{-1}$ \\
\hline$\tau_{F}$ & feeding threshold expressed in flagellate density & \#cells $\mathrm{L}^{-1}$ \\
\hline$\tau_{H}^{*}$ & lower threshold limit where a Hopf bifurcation will never occur & $\operatorname{mol~N~L}{ }^{-1}$ \\
\hline$V_{C}$ & ciliate (predator) biovolume & $\mu \mathrm{m}^{3}$ \\
\hline$V_{F}$ & flagellate (prey) biovolume & $\mu \mathrm{m}^{3}$ \\
\hline$v_{N}$ & nutrient searching rate & $\operatorname{mol~N~L}{ }^{-1} \mathrm{~d}^{-1}$ \\
\hline$v_{P}$ & prey searching rate & $\operatorname{mol~N~L}{ }^{-1} \mathrm{~d}^{-1}$ \\
\hline $\begin{array}{l}y_{P Z} \\
Z\end{array}$ & $\begin{array}{l}\text { yield of predator biomass on prey biomass, with } 0 \leq y_{P Z} \leq 1 \\
\text { predator biomass density }\end{array}$ & $\begin{array}{l}\operatorname{mol~} \mathrm{N} \mathrm{mol} \mathrm{N}^{-1} \\
\operatorname{mol~N~L}^{-1}\end{array}$ \\
\hline
\end{tabular}

The results in Figure 1 show that the Hopf bifurcation occurs at higher $N_{T}$ levels for higher threshold levels $\tau_{H}$. This suggests a stabilising effect of the feeding threshold. In order to study this effect further we calculated the two-parameter diagram where besides $N_{T}$ also $\tau$ varies. In the left panel of Figure 2 this diagram is shown. Note the logarithmic scale of the horizontal axis, which was needed to illustrate the asymptotic approach of the Hopf-curve towards a single threshold value at high nutrient loading. There appears to be an upper limit for the $\tau_{H}$ values when $\lim N_{T} \rightarrow \infty$. The simple expression for this limiting threshold with abundant enrichment, reads

$$
\tau_{H}^{*}=\lim _{N_{T} \rightarrow \infty} \tau_{H}=K_{P Z}\left(\frac{y_{P Z} I_{P Z}}{d_{Z}}-1\right)^{-2} .
$$


Rosenzweig-MacArthur model
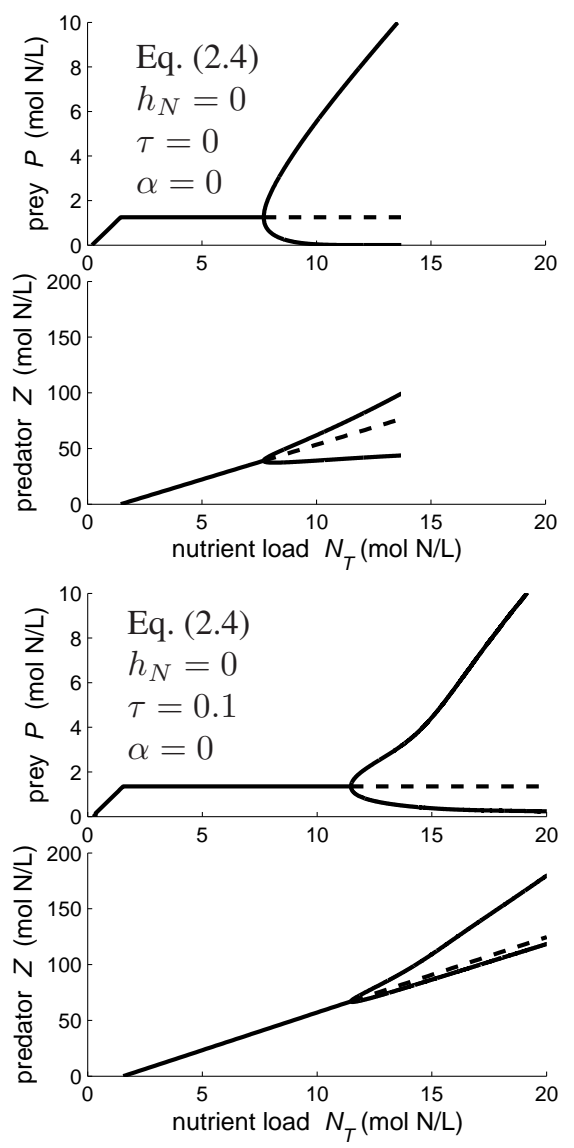
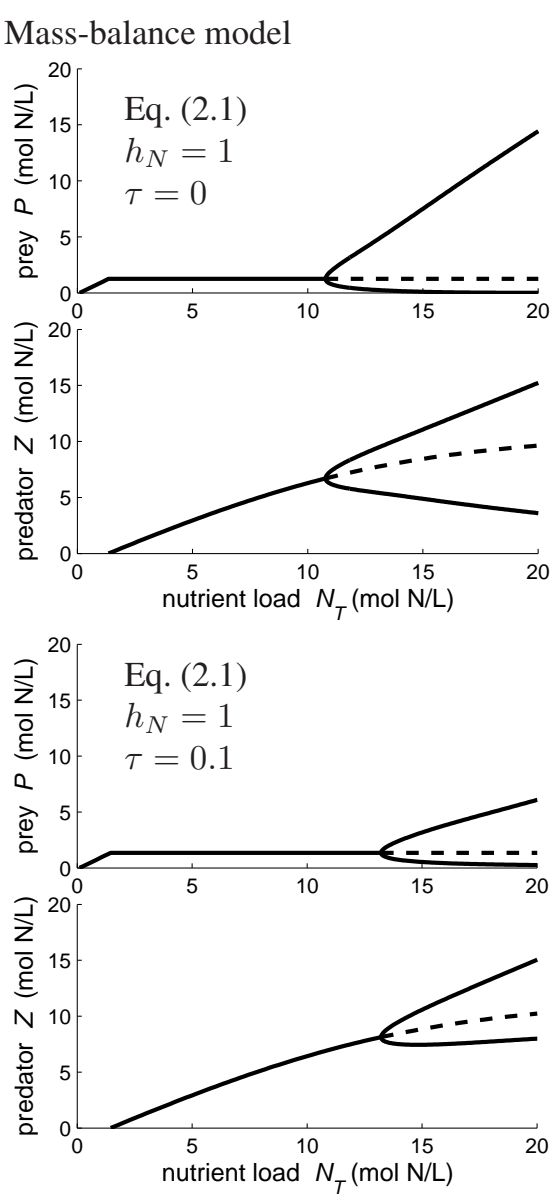

Figure 1: The biomasses of $P$ and $Z$ depend on the total nutrient load and the model used. Before the Hopf, the solid line denotes the equilibrium values, after the Hopf, the minima and maxima are denoted by the solid line and the equilibrium values by the dotted line. For the RM model, in the left hand panels, equilibrium densities were calculated with Eq. (2.4) with $\alpha$ set to zero. The simulations in the top-left panel are not continued due to very low densities of $P$ after $N_{T}=13.7$. For the mass-balance model, in the right side panels, equilibrium densities were based on Eq. (2.1) with a non-zero algal nutrient handling time. Note that the two models differ in scaling of the $Z$-axis. For both models the following parameters values were used: $d_{Z}=0.05, K_{P Z}=5.00$, $I_{P Z}=0.50, y_{P Z}=0.50, d_{P}=0.10, v_{N}=0.50$.

Observe that the value of $\tau_{H}^{*}$ solely depend on predator parameters. From Eq. (3.1) it can be observed that there is a lower threshold limit where a Hopf bifurcation will never occur for any nutrient enrichment. Therefore, the feeding threshold has a strong stabilising effect on nutrient enrichment, as defined in [31].

In order to get more insight into the disappearance of the Hopf bifurcation with feeding threshold values above $\tau_{H}^{*}$, we perform a phase-plane analysis of $Z$ vs. $P$. We zoom in on the Hopf bifurcation diagram of the RM model in Figure 2, this yields the left panel of Figure 3 in which the two-parameter bifurcation diagram is partially repeated. Next to this panel for two points on 
Rosenzweig-MacArthur model

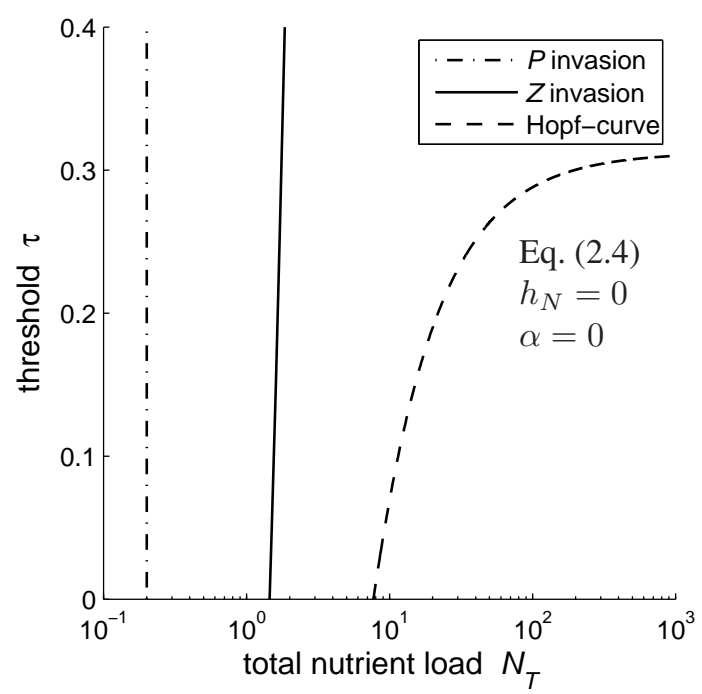

Mass-balance model

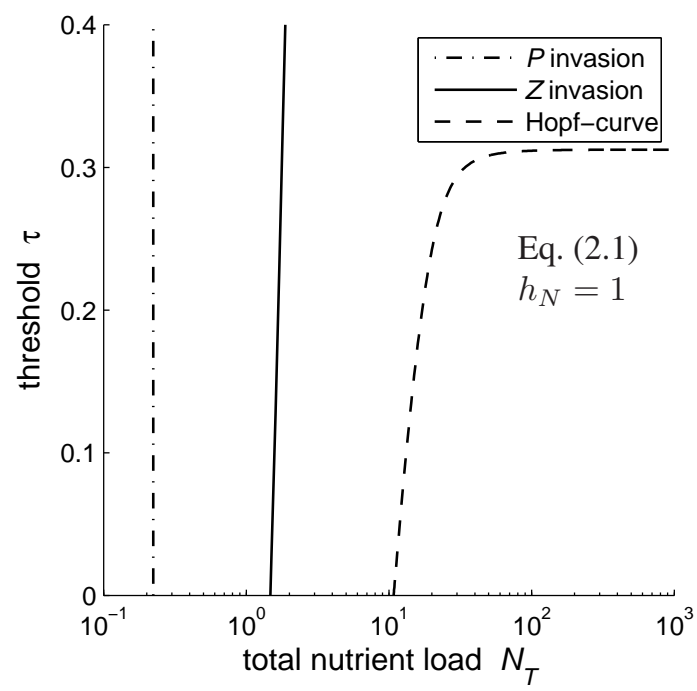

Figure 2: For each model the bifurcation diagram of $\tau$ vs. $N_{T}$ is shown. Each diagram contains the algal invasion threshold $(P)$, ciliate invasion threshold $(Z)$ and a Hopf-curve. For both models the following parameters values were used: $d_{Z}=0.05, K_{P Z}=5.00, I_{P Z}=0.50, y_{P Z}=0.50$, $d_{P}=0.10, v_{N}=0.50$.

the Hopf-curve the prey and predator zero-growth isoclines are drawn in the $Z$ vs. $P$ phase-space. Taking $d P / d t=0$ in Eq. (2.4a) with $\alpha=0$ and solving $Z$ and substituting $Z=g(P)$, we obtain $g(P)$ as the function for the prey zero-growth isocline ( $P$-isocline) given $P>0$

$$
Z=g(P)=\frac{r P\left(1-\frac{P}{K}\right)}{f(P)}
$$

To study the stability of the equilibrium $\left(E_{2}\right)$ formed at the intersection of the prey and predator isocline we derive the Jacobian and its trace and determinant. We obtain $\mathbf{J}_{11}=f(P) d g / d t$ and $\mathbf{J}_{22}=0$, from which we derive the eigenvalues $\lambda_{1,2}$

$$
\operatorname{Re} \lambda_{1,2}=\frac{1}{2} f(P) \frac{d g}{d P} .
$$

Hence for a Hopf bifurcation we require $d g / d P$ evaluated at the equilibrium equals to zero. Furthermore we have a stable equilibrium point when $d g / d P<0$ and an unstable equilibrium when $d g / d P>0$.

With $\tau=0$, we have the classical RM model, then the $P$-isocline is a parabola and the $Z$-isocline is a vertical line through the equilibrium value of prey population biomass. When $d g / d P=0$, then a Hopf-bifurcation occurs. This specific equilibrium is denoted by point I in Figure 3. Decreasing $N_{T}$ shifts the $g$-graph to the left and we have a new, stable equilibrium as 

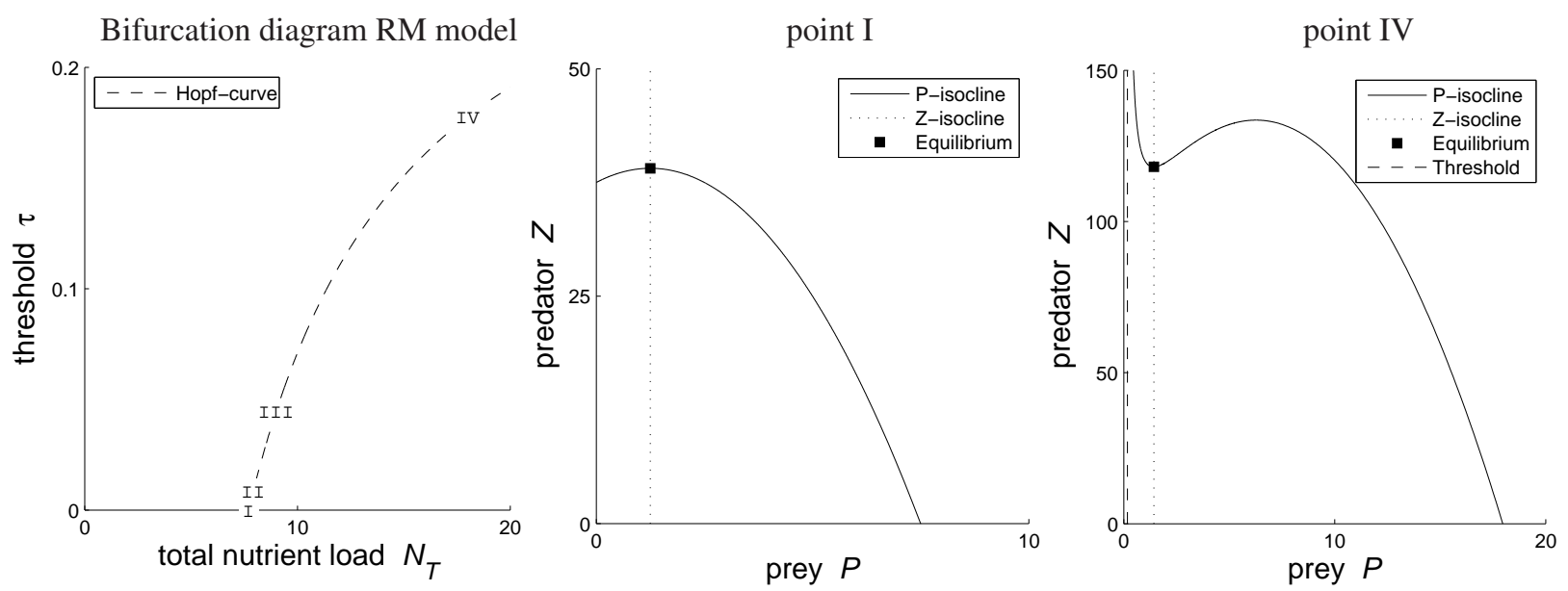

Figure 3: Different parameter values for nutrient load, $N_{T}$, and feeding threshold, $\tau$, affect the shape and location of the isoclines of the RM model (Eq. (2.4), $\alpha=0, h_{N}=0$ ). The left panel shows where in the bifurcation diagram the points I till IV are located on the Hopf-curve in respect to the values of $N_{T}$ and $\tau$. Point II and III are discussed in the text.Used parameter values: $K_{P Z}=5.00, I_{P Z}=0.50, y_{P Z}=0.50, d_{P}=0.10, v_{N}=0.50, d_{Z}=0.05$. $P$-isoclines for $P=0$ are omitted.

$d g / d P<0$. An increase in $N_{T}$ shifts the $g$-graph to the right where $d g / d P>0$ and the equilibrium becomes unstable. Observe that the place of the vertical $Z$-isocline does not depend on $N_{T}$.

For $\tau>0$ the shape of the $P$-isocline is not a parabola. Then $g$ has an asymptote at $P=\tau$ and crosses the $P$-axis at $P=N_{T}-d_{P} / v_{N}$. For the range of $\tau<P<N_{T}-d_{P} / v_{N}$ the slope of $g$ is (mostly) negative. For points II, III and IV on the Hopf bifurcation curve we have three different positive $\tau$-values and we also have $d g / d P=0$. For low $\tau$-values the equilibrium at the Hopf bifurcation point is on a maximum of the $P$-isocline function (Point II), but when increasing $\tau$ it becomes an inflection point (Point III) and then a minimum of the $P$-isocline function (Point IV). When the $\tau$-value approaches $\tau_{H}^{*}$ (see Eq. (3.1)) the minimum of function $g$ disappears via an inflection point, then $d g / d P$ is always negative. Thus for $\tau>\tau_{H}^{*}$, the equilibrium formed by the intersection of the vertical $Z$-isocline and the monotonously decreasing $P$-isocline is stable. This explains the occurrence of strong stability.

\subsection{The mass-balance model}

We performed a numerical bifurcation analyses of the mass-balance model Eq. (2.1). The results are shown in the right panels of Figure 1. Since the parameters have the same biological meaning in both the RM and MB model, we can directly compare the results for both models.

Figure 1 shows how the size of the limit cycles around the Hopf depends on the total nutrient 
load, $N_{T}$. In both models the feeding threshold delays the occurrence of the Hopf. Large amplitudes, occurring for $\tau=0.1$, mean potentially very low densities with an higher associated risk of extinction.

In Figure 2 the bifurcation diagrams of $\tau$ vs $N_{T}$ can be seen for both models, which contain: the invasion threshold of the algae, which is independent of $\tau$; the ciliate invasion threshold which is linearly proportional to $N_{T}$; and the Hopf-curve. These two-parameter diagrams show that there is an upper limit for the threshold for the occurrence of the Hopf bifurcation when $N_{T}$ is varied. We found for the MB model the same expression for $\tau_{H}^{*}$ as for the RM model, as given by Eq. (3.1).

In the previous section we defined $g$ from Eq. (3.2) as being the $P$-isocline of the RM model, here we define similarly $h$ as the $P$-isocline of the MB model (for $P>0$ ). For the following reasons we conclude that the behaviour of $h$ and $g$ is very similar. For $\tau=0$, both functions first increase and then decrease and cross the $P$-axis at the maximal attainable prey density in absence of predator. For $\tau>0$, both functions have an asymptote at $P=\tau$ and cross the $P$-axis at the same location as when $\tau=0$. As with the RM model, when $\tau>\tau_{H}^{*}$ the $P$-isocline is monotonously decreasing, which combined with the vertical $Z$-isocline causes strong stability.

\section{Applying the MB model on experimental data}

To illustrate the existence of a feeding threshold, we apply the MB model on experimental data consisting of dynamic growth curves of prey and predators from [33].

\subsection{Experimental setup and data description}

Prior to the feeding experiments, the predatory ciliate species Balanion planctonicum, Urotricha furcata and Urotricha farcta were maintained on the prey species Cryptomonas sp, an flagellated algae. All species are fresh water micro-organisms. The body-volumes of the predators are approximately 7 to 12 times larger than their prey. In each batch experiment the algal species is preyed upon by one ciliate species. In these closed systems, there is only gas-exchange while the medium is not refreshed.

The top panels of Figure 4, show the experimental data and the simulated growth curves (solid lines).

The top-left panel shows B. planctonicum feeding on Cryptomonas sp. and the top center panel shows $U$. furcata, also feeding on Cryptomonas $s p$. (vertical lines denote standard deviations, of which some are hidden by data markers.) In the control experiments, the algae were allowed to grow in $250 \mathrm{ml}$ culture flask until they reach a steady-state density. Thereafter density measurements were performed over time. In duplicates of the controls a single predatory ciliate species was added. This resulted directly in a decline of the algal population and in an increase of the predator population. Consequently, in these experiments the algae were reduced in density or went (nearly) extinct. A difference in feeding behaviour between B. planctonicum and $U$. furcata can be seen. The first predator exploits its resource completely, then it dies. While the latter predator does not totally consumes the prey population and settles for several months into a stable equilibrium 

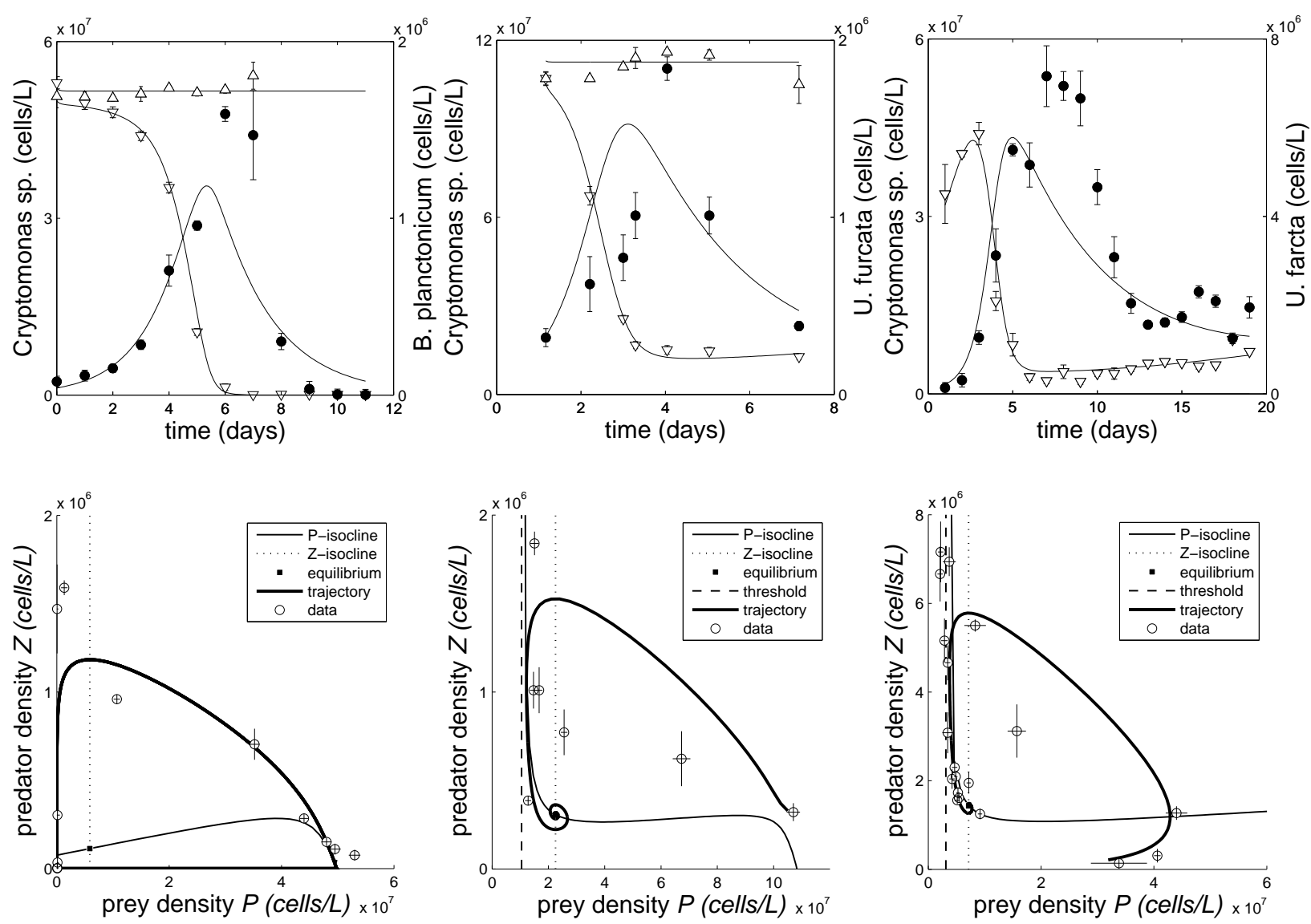

Figure 4: The mass-balance model based on Eq. (2.1) was fitted to experimental data from [33]. Each top panel shows a different predatory ciliate species feeding on the algal prey. $\triangle$ denotes cell densities of the prey species Cryptomonas $s p$. without predators present, $\nabla$ represents preyed Cryptomonas sp., • denotes cell densities of each ciliate species. Parameter values from Table 2 were used to construct simulated trajectories (solid lines in all panels) and the $P$ and $Z$-isoclines in the phase-spaces of $Z$ vs. $P$ (lower panels). $P$-isoclines for $P=0$ are omitted. Prey-predator datapoint combinations in the phase-space diagrams are represented by open circles with associated standard deviations represented by the extending lines.

(data not shown) [33]. The top-right panel shows Urotricha farcta feeding on Cryptomonas sp. No data is available for the control algal growth experiment. After 19 days the prey and predator seem to reach a stable equilibrium density.

Weisse et al. [33] used additional separate feeding experiments (semi-continuous cultures) in order to obtain the parameters for the Holling type-II functional response. Only for $U$. furcata the maximum ingestion rate, nutrient half-saturation constant and feeding threshold could be determined significantly. 


\subsection{Fitting method}

A weighted least-sum-of-squares method with weights equal to the inverse of the variance in the measurements was used. The sum of squares (SSQ) of prey and predator are summed into a dimensionless total SSQ, which is allowed as each measurement is weighted by its reciprocal standard deviation. A low sum-of-squares indicates a good fit. The data points values are assumed to be normally distributed. This makes this method identical to a maximum-likelihood method [20]. The SSQ is minimized with a Nelder-Mead's simplex method. The mean and covariance matrix (inverse of the Hessian matrix of the SSQ with respect to the parameters) of the estimated parameters are evaluated at the minimum SSQ-point [26]. Model fitting was done simultaneously on three data sets. Therefore, prey parameter values are identical for each data set.

\subsection{Fitting results}

The top panels of Figure 4, show the time evolution of the prey and predator populations. The lower panels show the $Z$ vs. $P$ phase-space diagrams. In each panel experimental data is shown with its associated standard deviations and the solid line represent the fitted trajectory. The lower left panel shows that the equilibrium for B. planctionicum is unstable as the trajectory of prey and predator converges to a periodic solution. The trajectory runs close to the $Z$ and $P$-axis, this indicates a risk of extinction for B. planctionicum. In the lower center and right panel for the two Urotricha species there exist a stable equilibrium to which the long-term trajectory converges.

Table 2 presents for each modelled species the estimated parameter-values and their uncertainties. Parameters included are initial densities, half-saturation constants, maximum feeding rates, feeding thresholds, yield factors and death rates.

We derived for $U$. furcata a feeding threshold value with $\tau_{F}=10500 \pm 923$ cells $/ \mathrm{ml}$. Furthermore, we found that the feeding threshold for $U$. farcta is a factor three lower compared to $U$. furcata. B. planctionicum was not found to have a feeding threshold.

\section{Discussion and conclusions}

A model property of the RM model is logistic growth of the prey in absence of the predator. To obtain the same property in the MB model we had to assume perfect and instantaneous nutrient recycling. We think this is warranted as Weisse et al. maintained for several months $U$. furcata cultures with Cryptomonas sp. even without exchanging the medium [33].

Aquatic ecosystem modelling with a feeding threshold for the trophic interactions is controversial. For instance in [30], the indiscriminate use of (constant) threshold values in models is criticized. In [19] it is claimed that their results suggest that any such thresholds should vary with prey quality. Nevertheless, in (some) ecotoxicological effect models feeding thresholds are taken into account, including AQUATOX [23, 24].

As part of the EU MODELKEY-project for assessing impact of pollutants on ecosystems[6], we designed a closed system with a single producer in order to measure the simultaneous effects of toxicant exposure and nutrient stress. A description of the experimental setup and obtained data 
Table 2: Mass-balance model (Eq. 2.1) fitted to data from Weisse et al. [33].

Fitted parameter values

\begin{tabular}{|c|c|c|c|c|c|c|c|}
\hline \multicolumn{8}{|c|}{ Prey species Cryptomonas sp. } \\
\hline Symbol & Value & $\mathrm{SD}^{*}$ & \multicolumn{5}{|l|}{ Units } \\
\hline$I_{N P}$ & $4.38 \cdot 10^{-1}$ ब & $3.90 \cdot 10^{-2}$ & \multicolumn{5}{|l|}{ day $^{-1}$} \\
\hline$d_{P}$ & $1.00 \cdot 10^{-1}$ & 0 & \multicolumn{5}{|l|}{ day $^{-1}$} \\
\hline$K_{N P}$ & $1.00 \cdot 10^{-6}$ & 0 & \multicolumn{5}{|l|}{$\operatorname{mol~N~L}{ }^{-1}$} \\
\hline \multicolumn{8}{|c|}{ constants and conversion factors } \\
\hline$V_{F}$ & $28 \underbrace{£_{1}}_{1}$ & 0 & \multicolumn{5}{|c|}{$\mu m^{3}$} \\
\hline$n_{F}$ & $7.50 \cdot 10^{-1 £_{2}}$ & 0 & \multicolumn{5}{|c|}{ pmol N cell ${ }^{-1}$} \\
\hline Figure & \multicolumn{2}{|l|}{$4 \mathrm{~A}$} & \multicolumn{2}{|c|}{$4 \mathrm{~B}$} & \multicolumn{3}{|l|}{$4 \mathrm{C}$} \\
\hline Predator & \multicolumn{2}{|c|}{ B. planctonicum } & \multicolumn{2}{|l|}{ U. furcata } & \multicolumn{3}{|l|}{ U. farcta } \\
\hline \multicolumn{8}{|c|}{ Ciliate model parameters } \\
\hline Symbol & Value & SD & Value & SD & Value & SD & Units \\
\hline$y_{P Z}$ & $2.18 \cdot 10^{-1}$ & $1.40 \cdot 10^{-2}$ & $1.82 \cdot 10^{-1}$ & $1.51 \cdot 10^{-2}$ & $2.00 \cdot 10^{-1}$ & 0 & - \\
\hline$I_{P Z}$ & $6.98 \cdot 10^{0} \dagger$ & $7.89 \cdot 10^{-1}$ & $1.56 \cdot 10^{1} \ddagger$ & $4.78 \cdot 10^{0}$ & $2.26 \cdot 10^{1 \S}$ & $1.05 \cdot 10^{1}$ & $\mathrm{~mol} \mathrm{~N}$ mol N${ }^{-1} \mathrm{~d}^{-1}$ \\
\hline$d_{Z}$ & $5.12 \cdot 10^{-1}$ & $2.94 \cdot 10^{-2}$ & $3.93 \cdot 10^{-1}$ & $2.53 \cdot 10^{-2}$ & $1.85 \cdot 10^{-1}$ & $4.74 \cdot 10^{-3}$ & $\mathrm{~d}^{-1}$ \\
\hline$K_{F C}$ & $1.16 \cdot 10^{7}$ & $4.30 \cdot 10^{6}$ & $7.49 \cdot 10^{7}$ & $3.76 \cdot 10^{7}$ & $9.46 \cdot 10^{7}$ & $5.47 \cdot 10^{7}$ & \#cells $\mathrm{L}^{-1}$ \\
\hline & 0 & 0 & $1.05 \cdot 10^{7}$ & $9.23 \cdot 10^{5}$ & $3.12 \cdot 10^{6}$ & $2.29 \cdot 10^{5}$ & \#cells $\mathrm{L}^{-1}$ \\
\hline \multicolumn{8}{|c|}{ constants and conversion factors** } \\
\hline$V_{C}$ & $2015^{£_{3}}$ & 0 & $3150^{£_{3}}$ & 0 & $3350^{£_{3}}$ & 0 & $\mu m^{3}$ \\
\hline$n_{C}$ & $5.40 \cdot 10^{0}$ & 0 & $8.44 \cdot 10^{0}$ & 0 & $1.37 \cdot 10^{0}$ & $1.01 \cdot 10^{-1}$ & pmol $\mathrm{N}$ cell ${ }^{-1}$ \\
\hline \multicolumn{8}{|c|}{ Initial conditions } \\
\hline$N(0)$ & 0 & 0 & 0 & 0 & $1.00 \cdot 10^{-3}$ & 0 & $\mathrm{~mol} \mathrm{~N} \mathrm{~L}^{-1}$ \\
\hline$F_{0}(0)$ & $5.20 \cdot 10^{7}$ & $2.27 \cdot 10^{5}$ & $1.13 \cdot 10^{8}$ & $2.76 \cdot 10^{5}$ & n.a. & n.a. & \#cells L $\mathrm{L}^{-1}$ \\
\hline$F_{C}(0)$ & $4.99 \cdot 10^{7}$ & $5.10 \cdot 10^{5}$ & $1.05 \cdot 10^{8}$ & $2.31 \cdot 10^{6}$ & $3.19 \cdot 10^{7}$ & $1.15 \cdot 10^{6}$ & \#cells $\mathrm{L}^{-1}$ \\
\hline$C(0)$ & $3.95 \cdot 10^{4}$ & $6.78 \cdot 10^{3}$ & $3.29 \cdot 10^{5}$ & $4.98 \cdot 10^{4}$ & $2.13 \cdot 10^{5}$ & $4.76 \cdot 10^{4}$ & \#cells $\mathrm{L}^{-1}$ \\
\hline \multicolumn{8}{|c|}{ Compound parameter values } \\
\hline \multicolumn{8}{|c|}{$\mu_{P Z}=y_{P Z} I_{P Z}-d_{Z}$} \\
\hline$\mu_{P Z}$ & $1.01 \cdot 10^{0}$ & - & $2.45 \cdot 10^{0}$ & - & $4.34 \cdot 10^{0}$ & - & $d^{-1}$ \\
\hline
\end{tabular}

*: A standard deviation (SD) of 0 means the variable or constant has an assumed nominal value. A dash means the value is based on the fitted parameter values. ${ }^{* *}$ : For conversion from cells/L to biomass N/L take $P(0)=n_{F} F(0)$, $Z(0)=n_{C} C(0), K_{P Z}=n_{F} K_{F C}$ and $\tau=n_{F} \tau_{F}$. We assumed $n_{C}=n_{F} V_{C} / V_{F}$, i.e. an identical amount of fmol $\mathrm{N} \mu \mathrm{m}^{-3}$ for both prey and predator. This relation was not used for $U$. farcta. ${ }^{\top}$ : Corresponds to $13.4 \mathrm{fmol} \mathrm{N}$ per cell per hour. ${ }^{£_{1}}$ : Based on [34]. ${ }^{£_{2}}$ : Based on [17]. ${ }^{£_{3}}$ : Based on [33]. ${ }^{\dagger}$ : Corresponds to $I_{F C}=2.09 \cdot 10^{0} \pm$ $2.37 \cdot 10^{-1}$ prey/ predator/ h. ${ }^{\ddagger}$ : Corresponds to $I_{F C}=7.31 \cdot 10^{0} \pm 2.24 \cdot 10^{0}$ prey/ predator/ h. ${ }^{\S}$ : Corresponds to $I_{F C}=1.72 \cdot 10^{0} \pm 8.13 \cdot 10^{-1}$ prey/ predator/ h (propagation of errors included).

can be found in [17]. We analysed these data successfully using a mass-balance model in [5]. The same experimental setup was used to investigate chemical stress and nutrient stress on a community with a producer and predator, respectively Cryptomonas $s p$. and $U$. furcata, with resulting data also presented in [17]. To analysing these results, we used a model with a Holling type-II functional response for predator-prey interaction. Without the inclusion of a feeding threshold the model would not fit the data and the prey population will incorrectly be driven to extinction.

The aim of the research presented in this article was twofold. Firstly to find out whether a feeding threshold for $U$. furcata (or a similar ciliate species) can be found in other data sets than 
[17] and secondly what its effect would be on the long term behaviour of these modelled systems.

The absence of a feeding threshold for both B. planctonicum and S. lacustris and the presence of a threshold for Histiobalantium bodamicum was reported in [21]. Using batch experiment data and the MB model, we found non-zero feeding thresholds for $U$. furcata and $U$. farcta but an absence of a feeding threshold for B. planctonicum. More specifically for $U$. furcata, we found a maximum ingestion rate of $I_{F C}=7.3 \pm 2.2$ cells/cell/h, a nutrient half-saturation constant of $K_{F C}=74900 \pm$ 37600 cells $/ \mathrm{ml}$ and a feeding threshold of $\tau_{F}=10500 \pm 923$ cells $/ \mathrm{ml}$. Weisse et al. [33] obtained from additional separate feeding experiments the Holling type-II functional response parameters for $U$. furcata, namely a maximum ingestion rate of $I_{F C}=5.3 \pm 0.7$ cells/cell/h, a nutrient halfsaturation constant of $K_{F C}=28200 \pm 8740 \mathrm{cells} / \mathrm{ml}$ and a feeding threshold of $\tau_{F}=13350 \pm 740$ cells $/ \mathrm{ml}$.

In the top center and right panels of Figure 4, the maximum decrease of the measured prey densities do not occur at the same moment in time moment as the maximum increase of the measured predator densities, i.e. for $U$. furcata and $U$. farcta, respectively. The MB model and the RM model both effectively have two variables, $P$ and $Z$, with $d Z / d t$ and $-d P / d t$ being mostly determined by the trophic interaction. Therefore, the simulated maximum decrease of the prey-curve is at the same time moment as the simulated maximum increase of the predator-curve. Thus, both models will never fit exactly on experimental data in which the maximum decline of the prey and the maximum increase of the predator do not coincide in time. This explains why the MB model does not capture the peaks of the ciliate densities at either the right moment or at the right density perfectly. Nevertheless, the fit is reasonable and justifies the use a feeding threshold. We used the MB model as it contains as few parameters and variables as possible while the biological meaning of the used parameters is retained.

We analysed the effect of a feeding threshold and enrichment on the stability of the MB and RM model. Both models are two dimensional systems in which the sole prey population suffers from intraspecific competition and a fixed part of that prey population is invulnerable to predation. The model properties of the RM model have been analysed earlier, mostly with the carrying capacity, $K$, as bifurcation parameter where increasing $K$ means enrichment. Here we analysed how stability depends on the two bifurcation parameters $N_{T}$ and $\tau$. Continuing $N_{T}$ affects both $r$ and $K$ indirectly, see Eq. (2.5), while normally $r$ and $K$ are treated as separate bifurcation parameters.

Previous studies of alternative models for the RM model implicate many potential mechanisms that can give stabilisation. These include predator-induced defence mechanisms, spatial heterogeneity and dormancy of the predators. The first two stabilizing effects work via predation-free prey populations who donate individuals to preyed populations which subsequently sustain the predators, this is classified as "donor-controlled" dynamics [2]. Dormancy is an adaptive response by the predator to a harsh environment, e.g. low prey density [16]. For copepods a feeding threshold was already known [22]. There the existence of a threshold was explained by assuming that the energy gained from captured food is too little compared to the energy spend on searching and capturing food. Therefore, not searching and not feeding is energetically more advantageous when food is limited.

When plotting $f(P)$ vs. $P$, the threshold shifts the intersection of the predator-prey functional response and the $P$-axis to the right of the origin. The lower center and right panels of Figure 4 
show how this shift in $f(P)$ cause the existence of an asymptote for the $P$-isocline in the phasespace of $Z$ vs. $P$. The presence of an asymptote makes the $P$-isocline a (mostly) decreasing function, with a decreasing function being an requirement for stability given a vertical $Z$-isocline. This sets the circumstances in which strong stability can occur when the threshold value becomes high enough.

Eq. (2.4b) always yields a vertical isocline for the predator for both the RM and MB model, with and without a feeding threshold. For both models the prey-isocline is a function of $P, g(P)$ and $h(P)$ respectively, forming a curve in the positive quadrant of the $P Z$-phase-space where for $P>\tau$ there is an unique $Z$. Therefore, the $P$-isocline will always intersect the $Z$-isocline only once. Consequently, in our MB model and the RM model formulation, a stable equilibrium is the global stable equilibrium.

We conclude that the bifurcation diagram for the RM and MB models with the same parameter values, are qualitatively identical, even when the feeding threshold was included, but they differ quantitatively. The feeding threshold has a strong stabilising effect in both model formulations.

The dynamics of low trophic levels will affect the dynamics of higher trophic levels and consequently a complete ecosystem. Therefore the inclusion (or exclusion) of feeding thresholds in models for the lowest trophic levels should carefully be contemplated.

\section{Acknowledgements}

We acknowledge financial support granted by the European Commission (Contract No 511237GOCE). The authors would like to thank Peter Abrams and one anonymous reviewer for useful comments and Markus Liebig for valuable discussions.

\section{References}

[1] P. A. Abrams, H. Matsuda. Prey adaptation as a cause of predator-prey cycles. Evolution, 51 (1997), 1742-1750.

[2] P. A. Abrams, C. J. Walters. Invulnerable prey and the paradox of enrichment. Ecology, 77 (1996), 1125-1133.

[3] J. R. Beddington. Mutual interference between parasites or predators and its effect on searching efficiency. J. Anim. Ecol., 44 (1975), 331-340.

[4] A. A. Berryman, B. A. Hawkins. The refuge as an integrating concept in ecology and evolution. Oikos, 115 (2006), No. 1, 192-196.

[5] D. Bontje, B. W. Kooi, M. Liebig, S. A. L. M. Kooijman. Modelling long-term ecotoxicological effects on an algal population under dynamic nutrient stress. Water Research, 43 (2009), 3292-3300. 
[6] W. Brack, J. Bakker, E. de Deckere, C Deerenberg, J. van Gils, M Hein, P Jurajda, S. Kooijman, M. Lamoree, S. Lek, M.-J. López de Alda, A. Marcomini, I. Muñoz, S. Rattei, H. Segner, K. Thomas, P. von der Ohe, B. Westrich, D. de Zwart, M. Schmitt-Jansen. Models for assessing and forecasting the impact of environmental key pollutants on freshwater and marine ecosystems and biodiversity. Environmental Science \& Pollution Research, 12 (2005), No. 5, 252-256.

[7] D. L. DeAngelis. Dynamics of Nutrient Cycling and Food Webs. Number 9 in Population and Community Biology series. Chapman \& Hall, London (1992).

[8] D. L. DeAngelis, R. A. Goldstein, R. V. O’Neill. A model for trophic interaction. Ecology, 56 (1975), 881-892.

[9] V. Grimm, C. Wissel. Babel, or the ecological stability discussions: an inventory and analysis of terminology and a guide for avoiding confusion. Oecologia, 109 (1997), 323-334.

[10] T. Gross, W. Ebenhöh, U. Feudel. Enrichment and foodchain stability: the impact of different forms of predator-prey interaction. J. Theor. Biol., 227 (2004), 349-358.

[11] V. A. A. Jansen. Regulation of predator-prey systems through spatial interactions: a possible solution to the paradox of enrichment. Oikos, 74 (1995), 384-390.

[12] K. L. Kirk. Enrichment can stabilize population dynamics: autotoxins and density depencence. Ecology, 79 (1998), 2456-2462.

[13] B. W. Kooi. Numerical bifurcation analysis of ecosystems in a spatially homogeneous environment. Acta Biotheoretica, 51 (2003), No. 3, 189-222.

[14] B. W. Kooi, J. C. Poggiale, P. Auger, S. A. L. M. Kooijman. Aggregation methods in food chains with nutrient recycling. Ecological Modelling, 157 (2002), No. 1, 69-86.

[15] M. Kretzschmar, R. M. Nisbet, E McCauley. A predator-prey model for zooplankton grazing on competing algal populations. Theor. Popul. Biol., 44 (1993), 32-66.

[16] M Kuwamura, T Nakazawa, T Ogawa. A minimum model of prey-predator system with dormancy of predators and the paradox of enrichment. Journal of Mathematical Biology, 58 (2009), No. 3, 459-479.

[17] M. Liebig, G. Schmidt, D. Bontje, B. W. Kooi, G. Streck, W. Traunspurger, T. Knacker. Direct and indirect effects of pollutants on algae and algivorous ciliates in an aquatic indoor microcosm. Aquatic Toxicology, 88 (2008), 102-110.

[18] Maplesoft. Maple. Maplesoft, Waterloo, Ontario, Canada (2003).

[19] A. Mitra, K. J. Flynn. Importance of interactions between food quality, quantity, and gut transit time on consumer feeding, growth, and trophic dynamics. The American Naturalist, 169 (2007), No. 5, 632-646. 
[20] A. M. Mood, F. A. Graybill, D. C. Boes. Introduction to the Theory of Statistics. McGrawHill, Inc., New York, $3^{\text {th }}$ edition (1974).

[21] H. Müller, A. Schlegel. Responses of three freshwater planktonic ciliates with different feeding modes to cryptophyte and diatom prey. Aquat. Microb. Ecol., 17 (1999), 49-60.

[22] M. M. Mullin, E. F. Stewart, F. J. Fuglister. Ingestion by planktonic grazers as a function of concentration of food. Limnol. Oceanogr., 20 (1975), 259-262.

[23] R. A. Park, J. S. Clough. Aquatox for windows: A modular fate and effects model for aquatic ecosystems. Technical Report 2, EPA (2004).

[24] R.A. Park, J. S. Clough, M. C. Wellman. Aquatox: Modeling environmental fate and ecological effects in aquatic ecosystems. Ecological Modelling, 213 (2008), 1-15.

[25] S. Petrovskii, B-L Li, H. Malchow. Transition to spatiotemporal chaos can resolve the paradox of enrichment. Ecol. Complex., 1 (2004), 37-47.

[26] H. W. Press, S. A. Teukolsky, W. T. Vetterling, B. P. Flannery. Numerical Recipes in C. Cambridge University Press, Cambridge, 2nd edition (1992).

[27] M. L. Rosenzweig, R. H. MacArthur. Graphical representation and stability conditions of predator-prey interactions. The American Naturalist, 97 (1963), 209-223.

[28] A. Saage, O. Vadstein, U. Sommer. Feeding behaviour of adult Centropages hamatus (Copepoda, Calanoida): Functional response and selective feeding experiments. Journal of Sea Research, 62 (2009), 16-21.

[29] M. Scheffer, R. J. De Boer. Implications of spatial heterogeneity for the paradox of enrichment. Ecology, 76 (1995), 2270-2277.

[30] S. L. Strom, C. B. Miller, B. W. Frost. What sets lower limits to phytoplankton stocks in high-nitrate, low-chlorophyll regions of the open ocean? Marine Ecology Progress Series, 193 (2000), 19-31.

[31] G. A. K. Van Voorn, D. Stiefs, T. Gross, B. W. Kooi, U. Feudel, S. A. L. M. Kooijman. Stabilization due to predator interference: comparison of different analysis approaches. Mathematical Biosciences and Engineering, 5 (2008), No. 3,:567-583.

[32] M. Vos, B. W. Kooi, D. L. DeAngelis, W. M. Mooij. Inducible defences and the paradox of enrichment. Oikos, 105 (2004), 471-480.

[33] T. Weisse, N. Karstens, V.C.L. Meyer, L. Janke, S. Lettner, K. Teichgräber. Niche separation in common prostome freshwater ciliates: the effect of food and temperature. Aquatic Microbial Ecology, 26 (2001),167-179. 
[34] T. Weisse, B Kirchhoff. Feeding of the heterotrophic freshwater dinoflagellate Peridiniopsis berolinense on cryptophytes: analysis by flow cytometry and electronic particle counting. Aquat. Microb. Ecol., 12 (1997), 153-164.

\section{Appendix}

Assume a closed ecosystem contains one species of producers, $P$, which capture energy from light but is effectively only limited in its growth by one type of nutrient, $N$, in the medium, e.g. phosphor or nitrogen containing compounds. These producers are preyed upon by predators, $Z$, via Holling type-II trophic interaction. The conversion of prey biomass into predator biomass is not $100 \%$ efficient, thus feaces and organic waste are produced and form together a pool of detritus, $M$. Biomass from deceased producers, predators and bacteria also add to this detritus. The bacteria, $B$, grow on detritus via Holling type-II with handling time $h_{M}$ and search rate $v_{M}$. Metabolic activities of the bacteria convert detritus into new bacterial biomass and freely available nutrients for the producers. This closes the nutrient loop. Detritus is converted with efficiency $y_{M B}$. The bacteria die with rate $d_{B}$, remaining parameters are explained in the text near Eq. (2.1). The above leads to the following ODE-system

$$
\begin{aligned}
\frac{d N}{d t} & =-P \frac{v_{N} N}{1+v_{N} h_{N} N}+\left(1-y_{B M}\right) B \frac{v_{M} M}{1+v_{M} h_{M} M} \\
\frac{d P}{d t} & =P\left(\frac{v_{N} N}{1+v_{N} h_{N} N}-d_{P}\right)-Z \frac{v_{P} P}{1+v_{P} h_{P} P} \\
\frac{d Z}{d t} & =Z\left(y_{P Z} \frac{v_{P} P}{1+v_{P} h_{P} P}-d_{Z}\right) \\
\frac{d B}{d t} & =B\left(y_{B M} \frac{v_{M} M}{1+v_{M} h_{M} M}-d_{B}\right) \\
\frac{d M}{d t} & =\left(1-y_{P Z}\right) Z \frac{v_{P} P}{1+v_{P} h_{P} P}+d_{P} P+d_{Z} Z+d_{B} B-B \frac{v_{M} M}{1+v_{M} h_{M} M} .
\end{aligned}
$$

Adding the masses of the degrading organisms and the dead organic material gives

$$
\begin{aligned}
\frac{d N}{d t} & =-P \frac{v_{N} N}{1+v_{N} h_{N} N}+\left(1-y_{B M}\right) B \frac{v_{M} M}{1+v_{M} h_{M} M}, \\
\frac{d P}{d t} & =P\left(\frac{v_{N} N}{1+v_{N} h_{N} N}-d_{P}\right)-Z \frac{v_{P} P}{1+v_{P} h_{P} P}, \\
\frac{d Z}{d t} & =Z\left(y_{P Z} \frac{v_{P} P}{1+v_{P} h_{P} P}-d_{Z}\right), \\
\frac{d(B+M)}{d t} & =\left(1-y_{P Z}\right) Z \frac{v_{P} P}{1+v_{P} h_{P} P}+d_{P} P+d_{Z} Z-\left(1-y_{B M}\right) B \frac{v_{M} M}{1+v_{M} h_{M} M} .
\end{aligned}
$$

The rate of change of $d(B+M) / d t$ depends on the biological rates related to feeding and death. Lets assume these rates are fast. This then allows for a quasi-steady state assumption with $d(B+M) / d t=0$, that yields Eq. (2.1). 